Article

\title{
Nutrient Intake through Childhood and Early Menarche Onset in Girls: Systematic Review and Meta-Analysis
}

\author{
Ngan Thi Kim Nguyen ${ }^{1}$, Hsien-Yu Fan ${ }^{2}$, Meng-Che Tsai ${ }^{3}$, Te-Hsuan Tung ${ }^{1}{ }^{\mathbb{D}}$, \\ Quynh Thi Vu Huynh ${ }^{4}$, Shih-Yi Huang ${ }^{1,5,6, * \mathbb{D}}$ and Yang Ching Chen $1,2,5,7, *$ (D) \\ 1 School of Nutrition and Health Sciences, Taipei Medical University, Taipei 110, Taiwan; \\ kimngan1702@gmail.com (N.T.K.N.); d07849004@ntu.edu.tw (T.-H.T.) \\ 2 Department of Family Medicine, Taipei Medical University Hospital, Taipei 110, Taiwan; \\ derossi83621@gmail.com \\ 3 Department of Pediatrics, National Cheng Kung University Hospital, College of Medicine, \\ National Cheng Kung University, Tainan 704, Taiwan; ache93@yahoo.com.tw \\ 4 Department of Pediatrics, Faculty of Medicine, The University of Medicine and Pharmacy at \\ Ho Chi Minh City, Ho Chi Minh City 700000, Vietnam; quynh.huynh@ump.edu.vn \\ 5 Graduate Institute of Metabolism and Obesity Sciences, Taipei Medical University, Taipei 110, Taiwan \\ 6 Nutrition Research Center, Taipei Medical University Hospital, Taipei 110, Taiwan \\ 7 Department of Family Medicine, School of Medicine, College of Medicine, Taipei Medical University, \\ Taipei 110, Taiwan \\ * Correspondence: sihuang@tmu.edu.tw (S.-Y.H.); melisa26@tmu.edu.tw (Y.C.C.); \\ Tel.: +886-2-2736-1661 (ext. 6543) (S.-Y.H.); +886-2-2737-2181 (ext. 3032) (Y.C.C.); \\ Fax: +886-2-2737-3112 (S.-Y.H.); +886-2-2738-9804 (Y.C.C.)
}

Received: 18 July 2020; Accepted: 20 August 2020; Published: 22 August 2020

\begin{abstract}
Among the genetic and environmental risk factors, nutrition plays a crucial role in determining the timing of puberty. Early menarche onset (EMO) is defined as when girls reach menarche onset at an age which is earlier than the mean/median age of menarche, between 12 and 13 years of age, according to individual ethnicity. The present study examined the association between nutrient intake in childhood and EMO risk in healthy girls by performing a systematic review and meta-analysis of prospective studies. We screened EMBASE, Cochrane Library, PubMed/MEDLINE, and Web of Science databases for 16 eligible studies with all medium-to-high quality scores ranging from 3 to 5 of 6 possible points with 10,884 subjects. Higher intakes of energy (risk ratio (RR) $=3.32$, $95 \%$ confidence interval $\left.(\mathrm{CI})=1.74-6.34, I^{2}=97 \%\right)$, and protein $(\mathrm{RR}=3.15,95 \% \mathrm{CI}=2.87-3.44$, $I^{2}=0 \%$ ) were associated with EMO risk. For each additional $1 \mathrm{~g} /$ day animal protein intake in childhood, the age at menarche was approximately two months earlier $\left(\beta=-0.13, I^{2}=55 \%\right)$, and high iron intake was associated with EMO (RR $\left.=1.20,95 \% \mathrm{CI}=1.03-1.40, I^{2}=0 \%\right)$. Polyunsaturated fatty acid (PUFAs) intake was associated with EMO risk with a dose-response effect (RR $=1.25$, $95 \% \mathrm{CI}=1.05-1.49, I^{2}=44 \%$ ). Girls with a high intake of fiber and monosaturated fatty acids (MUFAs) in childhood experienced later menarche onset $\left(R R=0.83,95 \% C I=0.69-1.00, I^{2}=31 \%\right.$; $R R=0.66$, $95 \% \mathrm{CI}=0.50-0.86, I^{2}=0 \%$, respectively). Thus, adherence to a high intake of animal proteins-, ironand PUFA-rich food diet makes girls more likely to have EMO, while a high intake of fiber- and MUFA-rich foods may protect girls from EMO. Further studies are expected to investigate the role of specific types of PUFAs and MUFAs on EMO to promote healthy sexual maturity in girls.
\end{abstract}

Keywords: puberty; menarche; sexual maturation; nutrient intake; childhood; meta-analysis 


\section{Introduction}

Since the late 19th century, early puberty onset, defined by a decreased mean age at menarche (AAM), has been noted as a global trend [1]. Menarche, the first menstrual period, is a widely used variable in epidemiological studies because it is a clear sign, is well-recalled, and occurs relatively late, between 12 and 13 years of age, and after the onset of breast development and the growth spurt in girls [2,3]. Individuals with earlier menarche onset (EMO) are at higher risk of hormone-related cancers [4,5], cardiovascular diseases [6], and all-cause mortality in later life [7]. The awareness of modifiable factors of EMO is thus critical to public health implications.

Of the genetic and environmental risk factors, nutrition plays a crucial role in determining the timing of puberty [1]. Caloric over-consumption may lead to EMO due to its contributions to the accumulation of body fat, which elevates leptin levels, a signal to the brain prompting puberty onset $[8,9]$. Therefore, sexual maturation is sensitive to nutritional regulation that requires the appropriate dietary intake for healthy pubertal growth. Investigations of nutrient intake in association with EMO might provide valuable information for further research on exploring the mechanism. Moreover, nutrient-based food groups or dietary pattern recommendations could be provided for children who have undergone pubertal growth.

Nutrient-based research serves to disseminate knowledge about essential nutrients that are important when making recommendations tailored to at-risk populations, such as EMO girls [10]. The fact that the associations of a prospective intake of energy [11,12], fat [13-15], fiber [16-18], and protein, including animal and vegetable protein [17,19] and EMO, remain controversial [1]. Although total fat intake in childhood has been reported to influence EMO [13,20], other findings have shown no clear evidence of the association between fat consumption and EMO [15,21]. High prepubertal isoflavone intake has been linked to delaying puberty onset in girls [22]. Foods containing high amounts of isoflavones are often rich in dietary fiber [23]. However, no associations between fiber and vegetable intake with menarche onset have been reported [15,19,22]. These opposing findings may have been due to heterogeneity in study design, methodological dietary assessment, and pubertal marker (growth spurt, thelarche, pubic hair, and menarche) [24]. In light of recent findings, to our knowledge, this systematic review and meta-analysis were firstly performed to investigate the effect of as many possible nutrient intakes on EMO among healthy girls.

\section{Materials and Methods}

We conducted a protocol for the systematic review, which was registered in the PROSPERO International Prospective Register of Systematic Reviews (https://www.crd.york.ac.uk/prospero, identifier CRD42020156430).

\subsection{Eligibility Criteria}

\subsubsection{Definitions of EMO and Non-EMO (NEMO)}

All the prepubertal girls recruited in each study were in a similar age range (Table S2). EMO was defined when girls reached menarche onset at an age that was earlier than mean/median AAM [25] (12-13 years of age [2,3]) according to individual ethnicity. For instance, the mean/median AAM of Canadian girls was 11.9 years [16,26], of American girls it was 12.5 years [13,21,27], of German girls it was 12.8 years [14,22,24], of English girls it was 12.8 years [19], of Tehranian girls it was 12.0 years [28], and of Australian girls it was 12.6 years [15]. Hence, our participants were divided into two groups according to pubertal outcomes: EMO and NEMO. The EMO group comprised girls who had menstruated before mean/median AAM in each study, meanwhile the NEMO group comprised girls who had not reached menarche. 


\subsubsection{Inclusion and Exclusion Criteria}

The PECOS (participants, exposure, comparison, outcomes, and study design) criteria (Table S1) was used to determine the eligibility of studies, the inclusion criteria was: (i) healthy prepubertal girls; (ii) nutrient intake in childhood assessed before menarche onset; (iii) nutrient intake assessment available for both EMO and NEMO groups; (iv) main outcomes were EMO and NEMO or AAM; and (v) prospective studies.

We excluded studies if (i) the children had any disease that affected pubertal development (e.g., congenital gonadal dysplasia or iodine deficiency disorder); (ii) the study was cross-sectional, a randomized control trial, an interventional study, a systematic review, or a meta-analysis; and (iii) puberty onset was defined by a growth spurt, thelarche, or pubic hair.

\subsection{Search Strategy}

The present study searched four electronic databases up to April 2020: EMBASE, Cochrane Library, PubMed/MEDLINE, and Web of Sciences. We used a medical subject heading terms and the free terms "menarche" or "menarcheal" or "puberty" or "pubertal" or "sexual maturation" or "timing of puberty" in combination with "diet" or "dietary intake" or "intake" or "nutrition" or "consumption." The results were limited by "case-control study" or "cohort analysis" or "comparative study" or "longitudinal study" or "observational study" or "prospective study" or "retrospective study." The search was related to human participants who were preschool children ( $2-5$ years), children (6-12 years), or adolescents (13-18 years). The search was also limited to English original articles.

One of our reviewers identified studies, and another reviewer screened the titles for eligibility. Both reviewers independently screened the abstracts and full-text articles. Disagreements were resolved by a third reviewer. All retrieved publications were entered into Endnote (v. 9.3, 2019, Clarivate).

\subsection{Data Extraction}

A standardized form was employed to extract data relating to the first author, publication year, country, population size, age at dietary assessment, dietary assessment method, nutrient intake, follow-up time, AAM, size of EMO and NEMO groups, confounders, and main findings. A standardized form was employed to extract data relating to the first author, publication year, country, population size, age at dietary assessment, dietary assessment method, nutrient intake, follow-up time, AAM, size of EMO and NEMO groups, confounders, and main findings.

\subsection{Quality Assessment of Evidence}

The Quality Assessment Tool for Systematic Reviews of Observational Studies (QATSO) was used [29]. Six questions could be answered with "yes" or "no" in four indicators: external validity (maximum of 1 point), reporting (maximum of 3 points), bias (maximum of 1 point), and confounders (maximum of 1 point). The total modified QATSO score could be up to 6 points ( 6 stars). Study quality can be divided into good (5-6 points), satisfactory (3-4 points), and poor ( $0-2$ points), representing a low, medium, and high risk of bias, respectively.

\subsection{Data Analysis and Statistical Methods}

Mean and standard deviation (SD) were computed from studies that provided the median, range, and sample size $[12,16,18,19]$ according to the method by Hozo et al. [30] We converted calories from megajoules and kilojoules to kilocalories $\left(1 \mathrm{kcal}=4.184 \mathrm{~kJ} ;=4.184 .10^{-3} \mathrm{MJ}\right)$. Calories expressed as $\mathrm{kcal} /$ height [28] were converted into calories ( $\mathrm{kcal}$ ) by referencing the World Health Organization child growth standard.

Though raw data queried from the authors were not available, we extracted the exact mean and SD of nutrient intake to assess the weighted mean difference (WMD) with $95 \%$ confidence interval (CI) between the EMO and NEMO groups without adjusting confounders. Sensitivity analyses were done 
by the adjusted risk ratio (RR) with $95 \%$ CI for a dichotomous outcome (EMO and NEMO), and the adjusted beta regression coefficient $(\beta)$ with standard error (SE) for a continuous outcome (AAM) were extracted from included studies that were controlled primarily by mother's AAM, parent education, age, height, body weight, and total EI (Table S1). Dose-response analyses were used to estimate the risk of different quartiles of nutrient intake in relation to EMO.

Pooled estimated results were calculated using the random-effect models to reflect variability in all studies. To assess between-study heterogeneity, Cochran's $Q$ test was used to obtain $p$ values and $I^{2}$ value. An $I^{2}>50 \%$ or a $p<0.05$ indicated heterogeneity across studies [31]. To detect publication bias, we used Egger's regression test, and $p<0.1$ has been recommended to indicate significant publication bias [32,33]. Meta-regression was performed to determine whether the baseline characteristics affected the heterogeneity across studies. Modifier factors were country (Canada, Chile, Colombia, England, Iran, Germany, Greece, Iran, Netherland, United States) [13,16,26,34], baseline body mass index (BMI) [12,13,19], age at dietary assessment (years old) [12,14,21], dietary measurement method (dietary record, food frequency questionnaire (FFQ) $[13,14,16,18,22,27,28]$, physical activity (hours/week) $[12,18,26]$, maternal AAM (years old) $[12,18,19,28]$, and socioeconomic status (high, middle, low) $[12,14,19,27,35]$. When $p<0.05$, subgroup analyses were applied.

All data were analyzed using the "meta" package in R software (v. 3.3.2, RStudio, Inc., Boston, MA, USA).

\section{Results}

\subsection{Study Selection}

4223 records were identified. After removing 1424 duplicates, we excluded 2519 records based on screening titles and abstracts. Of the remaining papers, 264 of 280 were removed after the full text had been assessed for eligibility. Finally, 16 longitudinal studies involving 10,884 girls were selected for systematic review and meta-analysis in the present study (Figure 1).

\subsection{Study Characteristics}

Dietary assessment was performed in early childhood (age $<8$ years) in 4 studies $[14,22,24]$ and in children aged $>8$ years in 11 studies [12,16-21,26-28,34] (Table 1). Dietary records were used in nine studies, the forms of which were 24 -h recall [28,34], 3- and 7-day records [16,17,26], and 3-day weighed records [14,24]. A food frequency questionnaire (FFQ) consisting of a semi-FFQ [12,18-21,27] or dietary history interview [13] was implemented in seven studies (Table 1).

Ten studies reported the total energy intake (EI) and macronutrient intake (carbohydrate, total protein, and total fat) in relation to EMO. A few of the studies considered the effects of monounsaturated fatty acids (MUFAs) [12,16,18,26,27], poly-unsaturated fatty acids (PUFAs) [12,16,18,19,26,27], micronutrients [16,19,26-28,34], animal protein [13-15,17,19,24], fiber [16-19,22,26,27], and isoflavones [22] on menarche onset. The mean difference in nutrient intake in childhood between the EMO and NEMO groups was analyzed through unadjusted testing $[12,18,19,27,28]$ and adjusted testing for age, mother's AAM, and EI $[16,26,27]$. The adjusted models were used to determine the association between EMO and either quartiles of nutrient intake $[16,18,20,21,26,27]$ or tertiles of nutrient intake [14,22]. Linear regression $[13,14,17]$ and logistic regression $[19,26,28]$ were used (Table 1).

\subsection{Quality Assessment}

Among the 16 selected studies, 8 studies $[12,16-19,22,26,28]$ were assessed to have low risk of bias (5-6 points) and 8 studies $[13-15,20,21,24,27,34]$ to have moderate risk of bias (3-4 points) (Table S2). No study was graded as having a high risk of bias (0-2 points). In addition, no significant publication bias was found (Table S3). 
Table 1. Characteristics of studies included in the systematic review.

\begin{tabular}{|c|c|c|c|c|c|c|c|c|}
\hline $\begin{array}{l}\text { Authors, Year, } \\
\text { Country }\end{array}$ & $\begin{array}{l}\text { Subjects(Mean } \pm \\
\text { SD/Age Range) } \\
\text { (Yrs) }\end{array}$ & $\begin{array}{l}\text { Dietary } \\
\text { Method }\end{array}$ & $\begin{array}{c}\text { Predictors } \\
\text { (Nutrient Intake) }\end{array}$ & $\begin{array}{l}\text { Follow-Up } \\
\text { (Yrs) }\end{array}$ & $\begin{array}{l}\text { Outcomes: AAM } \\
\text { (Mean } \pm \text { SD/Median, } \\
\text { Min, Max) (Yrs) }\end{array}$ & $\begin{array}{l}\text { Sample Size } \\
\text { Menarche: } \\
\text { Non-Menarche }\end{array}$ & Confounders & Main Findings \\
\hline $\begin{array}{l}\text { Kissinger and } \\
\text { Sanchez, 1987, } \\
\text { United States [34] }\end{array}$ & $\begin{array}{c}\text { Premenarcheal girls } \\
\text { aged 9-15 }\end{array}$ & $\begin{array}{l}\text { Multiple 24-h } \\
\text { recalls } \\
\text { Child reported }\end{array}$ & $\begin{array}{l}\text { EI, intakes of carbohydrate, } \\
\text { protein, fat, vitamins } \\
\text { Food groups }\end{array}$ & $>1$ & $\begin{array}{c}\text { AAM } \\
\text { Mother reported }\end{array}$ & $230: 770$ & $\begin{array}{l}\text { NA } \\
\text { NA }\end{array}$ & $\begin{array}{l}\text { (1) No mean difference data } \\
\text { between EMO and NEMO groups } \\
\text { (2) Upper quartile intake of } \\
\text { carbohydrate, } \\
\text { thiamine, and iron was linked to } \\
\text { 7-8-month later menarche } \\
\text { compared with the lowest quartile } \\
\text { ( } p<0.05)\end{array}$ \\
\hline $\begin{array}{l}\text { Moisan et al., } \\
\text { 1990a, Canada } \\
\text { [16] }\end{array}$ & $\begin{array}{c}2299 \text { Caucasian } \\
\text { girls aged } 11.1 \pm 0.6\end{array}$ & $\begin{array}{l}\text { 3-Day dietary } \\
\text { record } \\
\text { Child reported }\end{array}$ & $\begin{array}{l}\text { EI, intakes of macronutrients, } \\
\text { SFAs, MUFAs, PUFAs, } \\
\text { cholesterol, calcium, } \\
\text { phosphorus, iron, crude fiber, } \\
\text { and vitamins }\end{array}$ & 1.4 & $\begin{array}{c}12.1 \\
(10.1-14.7) \\
\text { Mother reported }\end{array}$ & 911:1388 & $\begin{array}{l}\text { Adjusted for age } \\
\text { and mother's AAM } \\
\text { Adjusted for } \\
\text { age and mother's } \\
\text { AAM }\end{array}$ & $\begin{array}{l}\text { (1) No differences in mean intake } \\
\text { of energy and macronutrients } \\
\text { between menarche and } \\
\text { premenarche groups }(p>0.05) \text {. } \\
\text { Higher intake of vitamin C was } \\
\text { linked to earlier AAM } \\
\text { (2) Girls with the highest quartile } \\
\text { of MUFA intake were likely to } \\
\text { have early menarche }\end{array}$ \\
\hline $\begin{array}{l}\text { Moisan et al., } \\
\text { 1990b, Canada } \\
\text { [26] }\end{array}$ & $\begin{array}{l}666 \text { Caucasia-n girls } \\
\text { aged } 9.5-12.5 \text { years }\end{array}$ & $\begin{array}{l}\text { 3-Day dietary } \\
\text { record } \\
\text { Child reported }\end{array}$ & $\begin{array}{l}\text { EI, intakes of macronutrients, } \\
\text { saturated FAs, MUFAs, } \\
\text { PUFAs, cholesterol, calcium, } \\
\text { phosphorus, iron, crude fiber, } \\
\text { and vitamins }\end{array}$ & 1 & $\begin{array}{c}11.4 \\
(10.1-13.3) \\
\text { Mother reported }\end{array}$ & 333:333 & $\begin{array}{l}\text { Adjusted for age } \\
\text { and EI } \\
\text { Adjusted for age } \\
\text { and EI }\end{array}$ & $\begin{array}{l}\text { (1) EMO girls consumed more } \\
\text { energy, carbohydrate, lipids, } \\
\text { MUFAs, iron, niacin, and vitamin } \\
\text { A than NEMO girls }(p<0.05) \\
\text { (2) EI was positively associated } \\
\text { with EMO in girls (OR = } 1.5, \\
95 \% \mathrm{CI}=0.9-2.3) \\
\text { Girls with the highest MUFA and } \\
\text { vitamin A intakes experienced } \\
\text { menarche later (OR }=0.6, \\
95 \% \mathrm{CI}=0.4-0.9 \text {; OR }=0.5, \\
95 \% \mathrm{CI}=0.3-0.8, \text { respectively) }\end{array}$ \\
\hline $\begin{array}{l}\text { Maclure et al., } \\
\text { 1991, United } \\
\text { States [27] }\end{array}$ & $\begin{array}{l}194 \text { girls, aged } \\
\quad 11.0 \pm 0.7\end{array}$ & $\begin{array}{l}\text { Semiquantitative } \\
\text { FFQ } \\
\text { Parent and child } \\
\text { reported }\end{array}$ & $\begin{array}{l}\text { Intakes of carbohydrate, } \\
\text { sucrose, fiber, protein, total fat, } \\
\text { FAs, cholesterol, and vitamins }\end{array}$ & 4 & $\begin{array}{c}12.5 \\
\text { Mother reported }\end{array}$ & $74: 120$ & $\begin{array}{c}\text { NA } \\
\text { Adjusted for } \\
\text { baseline EI, height, } \\
\text { and QI }\end{array}$ & $\begin{array}{c}\text { (1) Early menarche was associated } \\
\text { with higher intake of PUFAs, } \\
\text { vitamins A, C, and B12, carotene, } \\
\text { and retinol ( } p<0.05) \\
\text { (2) The highest intake of saturated } \\
\text { FAs, n-3 FAs, was associated with } \\
\text { later menarche than the reference } \\
\text { level (RR }=0.7,95 \% \mathrm{CI}=0.4-1.2 \text {; } \\
\mathrm{RR}=2.7,95 \% \mathrm{CI}=1.6-4.6 \text {, } \\
\text { respectively). The highest intake of } \\
\text { vitamin A was linked to AAM } \\
\text { compared with the reference } \\
\text { (RR }=1.3,95 \% \mathrm{CI}=0.7-2.3)\end{array}$ \\
\hline
\end{tabular}


Table 1. Cont

\begin{tabular}{|c|c|c|c|c|c|c|c|c|c|}
\hline $\begin{array}{l}\text { Authors, Year, } \\
\text { Country }\end{array}$ & $\begin{array}{c}\text { Subjects(Mean } \pm \\
\text { SD/Age Range) } \\
\text { (Yrs) }\end{array}$ & $\begin{array}{l}\text { Dietary } \\
\text { Method }\end{array}$ & \multicolumn{2}{|c|}{$\begin{array}{c}\text { Predictors } \\
\text { (Nutrient Intake) }\end{array}$} & $\begin{array}{l}\text { Follow-Up } \\
\quad \text { (Yrs) }\end{array}$ & $\begin{array}{l}\text { Outcomes: AAM } \\
\text { (Mean } \pm \text { SD/Median, } \\
\text { Min, Max) (Yrs) }\end{array}$ & $\begin{array}{c}\text { Sample Size } \\
\text { Menarche: } \\
\text { Non-Menarche }\end{array}$ & Confounders & Main Findings \\
\hline $\begin{array}{c}\text { de Ridder et al., } \\
\text { 1991, } \\
\text { Netherla-nds [17] }\end{array}$ & $\begin{array}{l}63 \text { girls aged } \\
9.6 \pm 0.04\end{array}$ & $\begin{array}{l}\text { 7-Day food } \\
\text { record } \\
\text { Child reported }\end{array}$ & \multicolumn{2}{|c|}{$\begin{array}{l}\text { EI, intakes of fiber, grain fiber, } \\
\text { vegetable fiber, protein fat, } \\
\text { carbohydrate }\end{array}$} & 3 & $\begin{array}{c}14.3 \\
\text { Child reported }\end{array}$ & $31: 29$ & $\begin{array}{l}\text { NA } \\
\text { Adjusted for EI, } \\
\text { height, dietary } \\
\text { methods, timing, } \\
\text { poly-saccharides }\end{array}$ & $\begin{array}{l}\text { (1) No mean difference data } \\
\text { between EMO and NEMO groups } \\
\text { (2) Vegetable protein and fiber } \\
\text { intakes were inversely associated } \\
\text { with earlier AAM ( } \beta=-3.3(1.5) \text {; } \\
\beta=-2.6 \text { (1.2), respectively) }\end{array}$ \\
\hline $\begin{array}{l}\text { Merzenich } \\
\text { et al., } \\
\text { 1993, } \\
\text { Germany [20] }\end{array}$ & $\underset{8-15}{261 \text { girls aged }}$ & $\begin{array}{l}\text { Semiquantitative } \\
\text { FFQ; } \\
\text { Parent and child } \\
\text { reported }\end{array}$ & \multicolumn{2}{|c|}{$\begin{array}{l}\text { Intakes of carbohydrate, } \\
\text { protein, and fat }\end{array}$} & 2 & $\begin{array}{l}9.7-15.6 \\
\text { Parent and child } \\
\text { reported }\end{array}$ & $94: 167$ & $\begin{array}{c}\text { NA } \\
\text { Adjusted by age } \\
\text { and total EI }\end{array}$ & $\begin{array}{l}\text { (1) No mean difference data } \\
\text { between EMO and NEMO groups } \\
\text { (2) The highest quartile accelerated } \\
\text { menarche onset }(\mathrm{RR}=2.1 \text {; } \\
95 \% \mathrm{CI}=1.1-4.0)\end{array}$ \\
\hline $\begin{array}{l}\text { Petridou et al., } \\
\text { 1996, Greece [12] }\end{array}$ & $\begin{array}{l}345 \text { girls, aged } \\
9-16\end{array}$ & $\begin{array}{c}\mathrm{FFQ} \\
\text { Child reported }\end{array}$ & \multicolumn{2}{|c|}{$\begin{array}{l}\text { EI, intakes of carbohydrate, } \\
\text { protein, saturated FAs, } \\
\text { MUFAs, and PUFAs }\end{array}$} & 1 & $\begin{array}{c}13 \\
(9.9-16.0) \\
\text { Mother reported }\end{array}$ & 166:179 & $\begin{array}{c}\text { NA } \\
\text { Adjusted for total EI }\end{array}$ & $\begin{array}{l}\text { (1) No mean difference data } \\
\text { between EMO and NEMO groups } \\
\text { (2) Girls with higher PUFA intake } \\
\text { reached menarche later than those } \\
\text { with the reference intake (RR= } \\
0.79,95 \% \mathrm{CI}=0.65-0.95)\end{array}$ \\
\hline $\begin{array}{l}\text { Koprowski et al. } \\
\text { 1999, United } \\
\text { States [21] }\end{array}$ & $\begin{array}{l}1378 \text { girls aged } \\
8-13\end{array}$ & $\begin{array}{l}\text { 7-Day FFQ } \\
\text { Child reported }\end{array}$ & \multicolumn{2}{|c|}{$\begin{array}{l}\text { Total EI and intakes of } \\
\text { carbohydrate, protein, and fat }\end{array}$} & 4 & $\begin{array}{l}\text { 9.7-14.8 } \\
\text { Parent reported }\end{array}$ & $464: 215$ & $\begin{array}{l}\text { NA } \\
\text { Adjusted for age at } \\
\text { dietary assessment, } \\
\text { height, QI, EI, and } \\
\text { ethnicity }\end{array}$ & $\begin{array}{l}\text { (1) No mean difference data } \\
\text { between EMO and NEMO groups } \\
\text { (2) Higher EI was inversely } \\
\text { associated with AAM ( } p \text { trend }< \\
\text { 0.05). Carbohydrate, protein, and } \\
\text { fat intakes were unrelated to AAM } \\
\quad(p<0.05)\end{array}$ \\
\hline $\begin{array}{l}\text { Berkey et al., 2000, } \\
\text { United States [13] }\end{array}$ & $\begin{array}{l}67 \text { Caucasian girls } \\
\text { aged } 3-5 \text { or } 6-8\end{array}$ & \multicolumn{2}{|c|}{$\begin{array}{l}\text { Dietary history } \\
\text { interview regarding the preceding } \\
6 \text { months } \\
\text { Mother reported }\end{array}$} & $\begin{array}{l}\text { EI, intakes } \\
\text { of animal } \\
\text { protein, } \\
\text { vegetable } \\
\text { protein, and } \\
\text { total fat }\end{array}$ & 4.83 and 8.83 & $\begin{array}{c}12.8 \pm 1.1 \\
\text { Medical records }\end{array}$ & NA & $\begin{array}{l}\text { NA } \\
\text { Adjusted for age } \\
\text { and EI }\end{array}$ & $\begin{array}{c}\text { (1) No mean difference data } \\
\text { between EMO and NEMO groups } \\
\text { (2) Vegetable protein intake at age } \\
\text { 3-5 years was linked to delayed } \\
\text { AAM }(\beta=2.19(0.91)) \text {. Animal } \\
\text { protein intake at age } 6-8 \text { years was } \\
\text { linked to earlier AAM }(\beta=-1.69 \\
(0.76))\end{array}$ \\
\hline $\begin{array}{l}\text { Koo et al., 2002, } \\
\text { Canada [18] }\end{array}$ & $\begin{array}{l}637 \text { girls aged } \\
\quad 9.7 \pm 1.2\end{array}$ & $\begin{array}{l}\text { Semiqu-antitative } \\
\text { FFQ } \\
\text { Mother } \\
\text { reported }\end{array}$ & \multicolumn{2}{|c|}{ Intakes of fiber, fat, and FAs } & 3 & $\begin{array}{c}13.6(8.5-15.6) \\
\text { Mother reported }\end{array}$ & $187: 402$ & $\begin{array}{c}\mathrm{NA} \\
\text { Adjusted for EI, age } \\
\text { at entry, } \\
\text { time-dependent age } \\
\text { at entry, body } \\
\text { weight, birth } \\
\text { weight, the } \\
\text { logarithm of EI, } \\
\text { mother's AAM, and } \\
\text { father's career }\end{array}$ & $\begin{array}{l}\text { (1) No mean difference data } \\
\text { between EMO and NEMO groups } \\
\text { (2) The fiber intake was negatively } \\
\text { associated with EMO (HR }=0.54 \text {, } \\
95 \% \mathrm{CI}=0.31-0.94) \text {. Increased } \\
\text { cellulose and animal fat intakes } \\
\text { were negatively associated with } \\
\text { menarche onset ( } p \text { trend }=0.009 \\
\text { and } 0.03 \text {, respectively). }\end{array}$ \\
\hline
\end{tabular}


Table 1. Cont.

\begin{tabular}{|c|c|c|c|c|c|c|c|c|}
\hline $\begin{array}{l}\text { Authors, Year, } \\
\text { Country }\end{array}$ & $\begin{array}{c}\text { Subjects(Mean } \pm \\
\text { SD/Age Range) } \\
\text { (Yrs) }\end{array}$ & $\begin{array}{l}\text { Dietary } \\
\text { Method }\end{array}$ & $\begin{array}{c}\text { Predictors } \\
\text { (Nutrient Intake) }\end{array}$ & $\begin{array}{l}\text { Follow-Up } \\
\quad(\text { Yrs })\end{array}$ & $\begin{array}{c}\text { Outcomes: AAM } \\
\text { (Mean } \pm \text { SD/Median, } \\
\text { Min, Max) (Yrs) }\end{array}$ & $\begin{array}{l}\text { Sample Size } \\
\text { Menarche: } \\
\text { Non-Menarche }\end{array}$ & Confounders & Main Findings \\
\hline $\begin{array}{c}\text { Cheng et al., 2010, } \\
\text { Germany [22] }\end{array}$ & $\begin{array}{l}119 \text { girls, aged } \\
\quad 7.2 \pm 1.0\end{array}$ & $\begin{array}{l}\text { 3-Day dietary } \\
\text { record }\end{array}$ & $\begin{array}{l}\text { Intakes of isoflavones and } \\
\text { dietary fiber at } 1 \text { and } 2 \text { years } \\
\text { before age at take-off }\end{array}$ & $1-2$ & $\begin{array}{c}12.6 \pm 1.0 \\
\text { to } \\
13.1 \pm 1.2 \\
\text { Parent and child } \\
\text { reported }\end{array}$ & 108:11 & $\begin{array}{c}\text { NA } \\
\text { Adjusted for } \\
\text { smoking, baseline } \\
\text { isoflavone intake, } \\
\text { and baseline EI } \\
\text { z-score } \\
\end{array}$ & $\begin{array}{l}\text { (1) No mean difference data } \\
\text { between EMO and NEMO groups } \\
\text { (2) Fiber and isoflavone intakes } \\
\text { were not associated with AAM in } \\
\text { the unadjusted model or adjusted } \\
\text { model ( } p \text { _trend }=0.4)\end{array}$ \\
\hline $\begin{array}{c}\text { Gunther et al., } \\
\text { 2010, Germany } \\
\text { [14] }\end{array}$ & $\begin{array}{l}112 \text { children (57 } \\
\text { girls, } 50.9 \% \text { ) }\end{array}$ & $\begin{array}{l}\text { 3-Day weighed } \\
\text { dietary record } \\
\text { for girls aged } \\
\text { 3-4 or 5-6 years } \\
\text { Parent reported }\end{array}$ & $\begin{array}{l}\text { Intakes of protein, animal } \\
\text { protein, and vegetable protein }\end{array}$ & $\begin{array}{l}\text { Average }=9.3 \\
\quad \text { and } 7.3\end{array}$ & $12.8 \pm 1.2$ & 47:10 & $\begin{array}{l}\text { NA } \\
\text { Adjusted for sex, EI, } \\
\text { breastfeeding, birth } \\
\text { year, and father's } \\
\text { university degree }\end{array}$ & $\begin{array}{l}\text { (1) No mean difference data } \\
\text { between EMO and NEMO groups } \\
\text { (2) Higher animal protein intake at } \\
5-6 \text { years was related to earlier } \\
\text { menarche ( } p \text { trend }=0.02) . \\
\text { Children with higher vegetable } \\
\text { protein intake experienced later } \\
\text { menarche }(p \text { trend }=0.02-0.03)\end{array}$ \\
\hline $\begin{array}{c}\text { Remer et al., 2010, } \\
\text { Germany [24] }\end{array}$ & $\begin{array}{l}109 \text { German } \\
\text { children (55 girls) }\end{array}$ & $\begin{array}{l}\text { 3-Day weighed } \\
\text { dietary record } \\
\text { Child reported }\end{array}$ & Intake of animal protein & $\begin{array}{c}1 \text { and } 2 \\
\text { before ATO }\end{array}$ & $\begin{array}{l}13.1 \pm 0.8 \\
\text { Parent and child } \\
\text { reported }\end{array}$ & NA & $\begin{array}{l}\text { NA } \\
\text { Adjusted for sex, EI, } \\
\text { fat mass index, } \\
\text { gestational age, } \\
\text { birth weight, } \\
\text { breastfeeding, and } \\
\text { maternal } \\
\text { overweight } \\
\end{array}$ & $\begin{array}{l}\text { (1) No mean difference data } \\
\text { between EMO and NEMO groups } \\
\text { (2) Animal protein intake was } \\
\text { negatively associated with AAM } \\
(p=0.07)\end{array}$ \\
\hline $\begin{array}{l}\text { Roger et al., 2010, } \\
\text { England [19] }\end{array}$ & $\begin{array}{c}3298 \text { girls aged } \\
12.9 \pm 0.2\end{array}$ & $\begin{array}{l}\text { FFQ at ages } 3 \\
\text { and } 7 \text { years. } \\
\text { 3-Day weight } \\
\text { dietary record at } \\
\text { age 10-11 years, } \\
\text { Child reported }\end{array}$ & $\begin{array}{l}\text { EI, intakes of total fat, } \\
\text { saturated FAs, MUFAs, } \\
\text { PUFAs, starch, sugar, total } \\
\text { protein, animal protein, } \\
\text { vegetable protein, fiber, } \\
\text { vitamins }\end{array}$ & 3.5 & $\begin{array}{c}12.8 \pm 0.2 \\
\text { Clinic reported }\end{array}$ & 951:1419 & $\begin{array}{l}\text { NA } \\
\text { Adjusted for BMI } \\
\text { and height at the } \\
\text { time of diet } \\
\text { measurement }\end{array}$ & $\begin{array}{c}\text { (1) At } 3 \text { years, protein, animal } \\
\text { protein, and carotene intakes were } \\
\text { higher in girls with EMO }(p<0.05) \text {. } \\
\text { At } 7 \text { years, PUFA, protein, animal } \\
\text { protein, and Zn intakes were } \\
\text { higher in girls with EMO } \\
(p=0.019) \text {. At } 10 \text { years, EI was } \\
\text { higher in girls with EMO } \\
\text { ( } p=0.002) \\
\text { (2) At aged } 3 \text { and } 7 \text { years, protein, } \\
\text { animal protein PUFA intakes were } \\
\text { positively associated with EMO, } \\
\text { but not at } 10 \text { years of age }\end{array}$ \\
\hline
\end{tabular}


Table 1. Cont.

\begin{tabular}{|c|c|c|c|c|c|c|c|c|}
\hline $\begin{array}{l}\text { Authors, Year, } \\
\text { Country }\end{array}$ & $\begin{array}{c}\text { Subjects(Mean } \pm \\
\text { SD/Age Range) } \\
\text { (Yrs) }\end{array}$ & $\begin{array}{l}\text { Dietary } \\
\text { Method }\end{array}$ & $\begin{array}{c}\text { Predictors } \\
\text { (Nutrient Intake) }\end{array}$ & $\begin{array}{l}\text { Follow-Up } \\
\text { (Yrs) }\end{array}$ & $\begin{array}{c}\text { Outcomes: AAM } \\
\text { (Mean } \pm \text { SD/Median, } \\
\text { Min, Max) (Yrs) }\end{array}$ & $\begin{array}{c}\text { Sample Size } \\
\text { Menarche: } \\
\text { Non-Menarche }\end{array}$ & Confounders & Main Findings \\
\hline $\begin{array}{l}\text { Tehrani et al., } \\
\text { 2013, Iran [28] }\end{array}$ & $\begin{array}{l}134 \text { prepubertal } \\
\text { girls aged } \\
8.9 \pm 2.4\end{array}$ & $\begin{array}{l}\text { 24-h Dietary } \\
\text { recalls } \\
\text { Child reported }\end{array}$ & $\begin{array}{l}\text { EI, intakes of carbohydrate, } \\
\text { fat, protein, magnesium, } \\
\text { phosphorus, } \\
\text { milk, yogurt, and cheese }\end{array}$ & $\begin{array}{l}\text { Medi-an } \\
\text { follow-up } \\
\quad=6.5\end{array}$ & $\begin{array}{c}12.7 \pm 1.3 \\
\text { Child reported }\end{array}$ & $60: 74$ & $\begin{array}{c}\text { NA } \\
\text { Adjusted for EI, } \\
\text { protein intake, the } \\
\text { interval between } \\
\text { the age at study } \\
\text { initiation and the } \\
\text { AAM, and mother's } \\
\text { AAM }\end{array}$ & $\begin{array}{c}\text { (1) No significant differences in EI } \\
\text { or carbohydrate, protein, or total } \\
\text { fat intake between EMO and } \\
\text { NEMO groups }(p<0.05) \\
(2) \text { Girls with EMO had higher } \\
\text { intakes of calcium }(\mathrm{OR}=3.20, \\
95 \% \mathrm{CI}=1.39-7.42), \text { magnesium } \\
(\mathrm{OR}=2.43,95 \% \mathrm{CI}=1.12-5.27) \\
\text { and phosphorus }(\mathrm{OR}=3.37 \\
95 \% \mathrm{CI}=1.44-7.87) \text { than girls with } \\
\text { NEMO. }\end{array}$ \\
\hline $\begin{array}{c}\text { Cheng et al., 2019, } \\
\text { Australia } \\
\text { [15] }\end{array}$ & $\begin{array}{l}142 \text { prepuber-tal } \\
\text { children aged } 8\end{array}$ & $\begin{array}{l}\text { 3-Day food } \\
\text { record } \\
\text { Parent and child } \\
\text { reported }\end{array}$ & $\begin{array}{l}\text { EI, intakes of carbohydrate, } \\
\text { fat, total protein, and animal } \\
\text { protein }\end{array}$ & $5-8$ & $\begin{array}{c}12.6 \pm 1.0 \\
\text { Child reported }\end{array}$ & $92: 50$ & $\begin{array}{c}\text { NA } \\
\text { Adjusted for total } \\
\text { energy, birth } \\
\text { weight, height, } \\
\text { zBMI, and mother's } \\
\text { occupation }\end{array}$ & $\begin{array}{l}\text { (1) No mean difference data } \\
\text { between EMO and NEMO groups } \\
\text { (2) Lower absolute }(p=0.04) \text { and } \\
\text { energy-adjusted ( } p=0.03 \text { ) protein } \\
\text { intake was linked to EMO. Lower } \\
\text { dietary protein (relative to } \\
\text { carbohydrate and fat) intake } \\
\text { consistently predicted EMO. } \\
\text { Animal protein intake was not } \\
\text { associated with menarche onset }\end{array}$ \\
\hline
\end{tabular}

Definition of abbreviations: AAM, age at menarche; BMI, body mass index; EMO, earlier menarche onset; EI, energy intake; FAs, fatty acids; FFQ, food frequency questionnaire; NA, not applicable; NEMO, non-early menarche onset; PUFA, polyunsaturated fatty acid; QI, Quetelet's index; RR, relative risk; SD, standard deviation; zBMI, z-score BMI. 


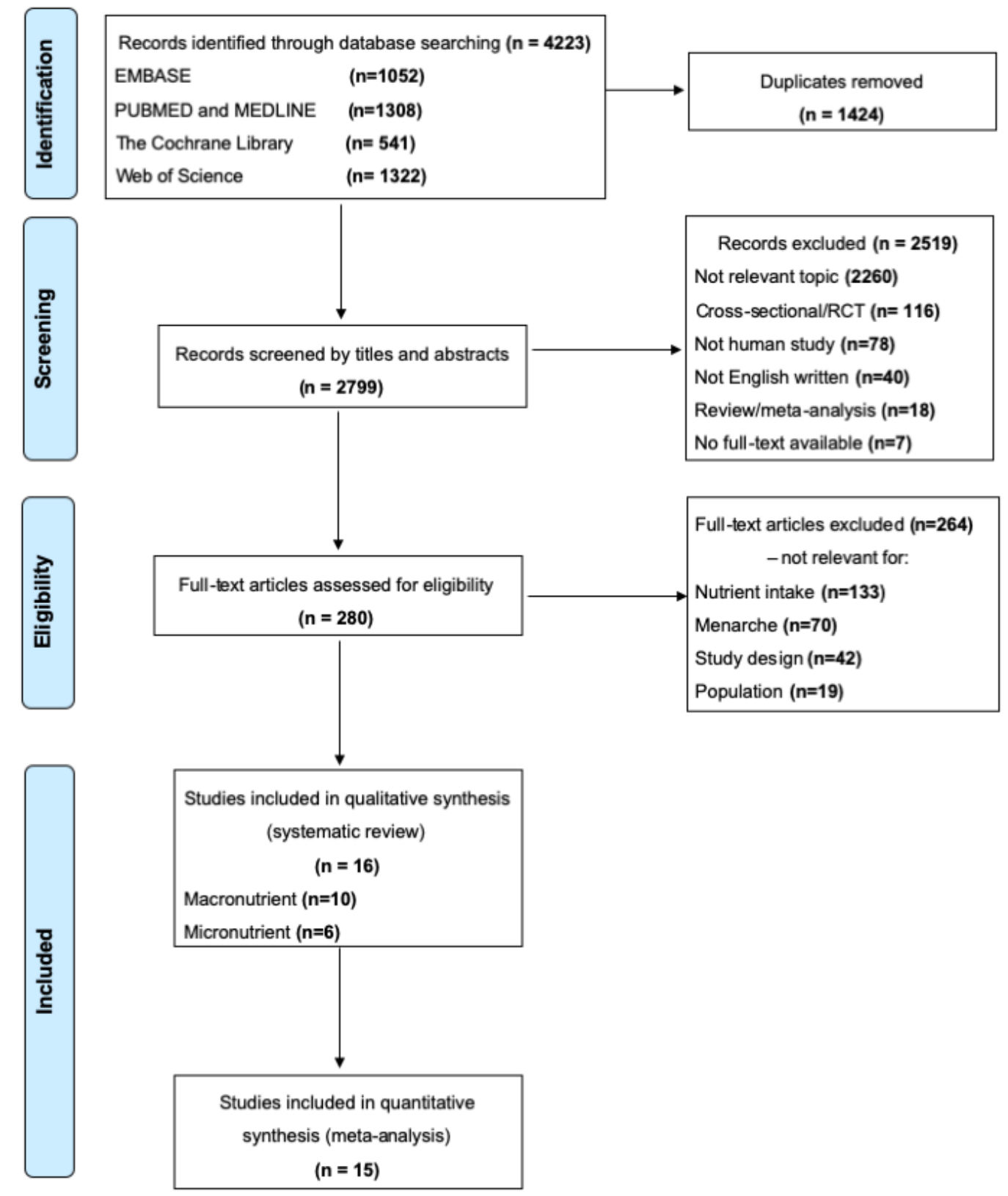

Figure 1. Study flow chart. Definition of abbreviations: EMBASE, Elservier database; RCT, randomized controlled trial.

\subsection{Meta-Analysis, Publication Bias, and Sensitivity Analysis}

\subsubsection{Association between Total EI and EMO}

Girls with EMO had a higher WMD of total EI than girls with NEMO with the existence of heterogeneity $\left(I^{2}=68 \%, p<0.01\right.$, Table 2$)$. Given that the meta-regression identified a possible effect of baseline BMI on the EI-EMO relationship (Table S4), we then performed subgroup analysis. For girls with $\mathrm{BMI} \geq 18.5 \mathrm{~kg} / \mathrm{m}^{2}$, the WMD of EI remained significantly higher in the girls with EMO than in the girls with NEMO (WMD $=68.1 \mathrm{kcal} / \mathrm{day}, 95 \% \mathrm{CI}=18.0$ to $118.1 \mathrm{kcal} / \mathrm{day}$, Figure 2$)$. Additional analysis showed that the higher EI was positively associated with EMO risk ( $\mathrm{RR}=3.3,95 \% \mathrm{CI}=1.7$ to 6.3; Figure 3a). 
Table 2. Mean differences in nutrient intake between girls with EMO and NEMO.

\begin{tabular}{|c|c|c|c|c|c|}
\hline \multirow{2}{*}{$\begin{array}{c}\text { Energy and Nutrient } \\
\text { Intake }\end{array}$} & \multirow{2}{*}{ EMO (N) } & \multirow{2}{*}{ NEMO (N) } & \multicolumn{2}{|c|}{ Weighted mean Difference (WMD, 95\% CI) } & \multirow{2}{*}{$I^{2}$} \\
\hline & & & Fixed Effect & Random Effect & \\
\hline Energy (kcal/day) & 2017 & 3230 & $60.2(40.9,79.6)$ * & $51.7(-1.1,104.5)$ & $68 \%$ * \\
\hline Carbohydrate (g/day) & 1398 & 2163 & $-0.1(-3.7,3.5)$ & $-2.8(-22.2,16.7)$ & $92 \%$ \\
\hline Carbohydrate (\% energy) & 1398 & 2163 & $-0.1(-3.8,0.8)$ & $-0.1(-3.8,0.8)$ & $0 \%$ \\
\hline Total fiber (g/day) & 1172 & 1910 & $0.0(-0.1,0.1)$ & $0.0(-0.1,0.1)$ & $0 \%$ \\
\hline Protein (g/day) & 2349 & 3582 & $0.9(0.3,1.5)$ * & $0.2(-1.4,1.8)$ & $62 \%$ * \\
\hline Protein (\% energy) & 2349 & 3582 & $0.2(0.1,0.3) *$ & $0.04(-0.3,0.3)$ & $56 \%$ \\
\hline Fat (g/day) & 1398 & 2163 & $1.2(0.02,2.4) *$ & $1.8(-0.7,4.2)$ & $34 \%$ \\
\hline Fat (\% energy) & 1398 & 2163 & $-0.8(-1.1,-0.5) *$ & $-0.03(-1.0,0.9)$ & $73 \%$ * \\
\hline Saturated FAs (g/day) & 1338 & 2089 & $0.2(-0.4,0.8)$ & $0.3(-1.4,2.1)$ & $60 \%$ \\
\hline MUFAs (g/day) & 1338 & 2089 & $0.5(0.04,1.0)$ * & $0.5(-0.3,1.4)$ & $18 \%$ \\
\hline PUFAs (g/day) & 2289 & 3508 & $0.4(0.2,0.6)$ * & $0.5(0.2,0.8)$ * & $21 \%$ \\
\hline Cholesterol (g/day) & 1172 & 1910 & $1.9(-4.3,8.1)$ & $1.9(4.3,8.1)$ & $0 \%$ \\
\hline Calcium (mg/day) & 1404 & 1649 & $-2.4(-31.5,26.7)$ & $-2.4(-31.5,26.7)$ & $0 \%$ \\
\hline Phosphate (mg/day) & 971 & 1462 & $8.9(-17.5,35.3)$ & $8.9(-17.5,35.3)$ & $0 \%$ \\
\hline Magnesium (mg/day) & 679 & 1141 & $3.1(0.3,5.9) *$ & $3.1(0.3,5.9) *$ & $0 \%$ \\
\hline Iron (mg/day) & 911 & 1388 & $0.2(-0.1 ; 0.4)$ & NA & NA \\
\hline Vitamin B1 (mg/day) & 985 & 1508 & $0.0(-0.03,0.03)$ & $0.0(-0.03,0.03)$ & $0 \% *$ \\
\hline Riboflavine ( $\mu \mathrm{g} /$ day) & 985 & 1508 & $-1.0(-1.0,-0.9) *$ & $-0.4(-1.6,0.7)$ & $96 \%$ * \\
\hline Vitamin B3 ( $\mu \mathrm{g} /$ day) & 985 & 1508 & $0.2(-0.3,0.6)$ & $0.2(-0.3,0.6)$ & $0 \%$ \\
\hline Vitamin B6 (mg/day) & 985 & 1508 & $0.0(-0.03,0.03)$ & $0.0(-0.03,0.03)$ & $0 \%$ \\
\hline Vitamin B9 ( $\mu \mathrm{g} /$ day) & 985 & 1508 & $-0.7(-7.8,6.4)$ & $-0.7(-7.8,6.4)$ & $0 \%$ \\
\hline Vitamin B12 ( $\mu \mathrm{g} /$ day) & 985 & 1508 & $0.01(-0.1,0.1)$ & $0.2(-0.4,0.8)$ & $59 \%$ \\
\hline Vitamin C (mg/day) & 1418 & 1695 & $5.4(1.1,9.7) *$ & $8.6(-9.7,26.9)$ & $23 \%$ \\
\hline Vitamin A (IU/day) & 1418 & 1695 & $-83.8(-218.3,50.7)$ & $1476.9(-2262.5,5261.4)$ & $81 \%$ * \\
\hline Carotene ( $\mu \mathrm{g} /$ day) & 1068 & 1600 & $78.1(13.9,142.4) *$ & $744.7(-992.9,2482.2)$ & $72 \%$ \\
\hline Vitamin D (IU/day) & 985 & 1508 & $-0.5(-10.4,9.4)$ & $-0.5(-10.4,9.4)$ & $0 \%$ \\
\hline Vitamin E (mg/day) & 985 & 1508 & $0.1(-0.1,0.3)$ & $0.1(-0.1,0.3)$ & $0 \%$ \\
\hline
\end{tabular}

${ }^{*} p<0.05$. Definition of abbreviations: CI, confidence interval; EMO, earlier menarche onset; FAs, fatty acids; MUFAs, monounsaturated fatty acids; NA, not applicable; NEMO, non-early menarche onset; PUFAs, polyunsaturated fatty acids; WMD, weighted mean difference.

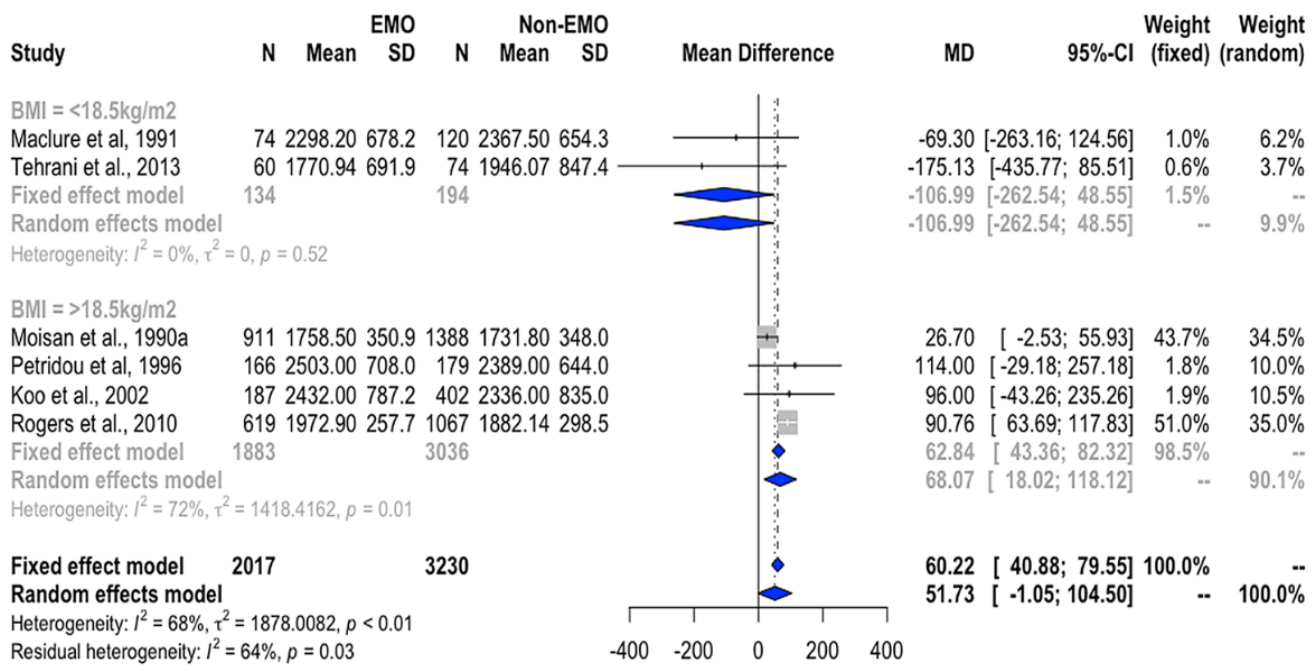

Figure 2. Forest plot of WMD of EI between EMO and NEMO groups stratified by BMI $<18.5 \mathrm{~kg} / \mathrm{m}^{2}$ and $\mathrm{BMI} \geq 18.5 \mathrm{~kg} / \mathrm{m}^{2}$. Definition of abbreviations: EMO, earlier menarche onset; NEMO, non-early menarche onset; $\mathrm{MD}$, mean difference; $\mathrm{WMD}$, weighted mean difference. 
(a) Total El (kcal/day)

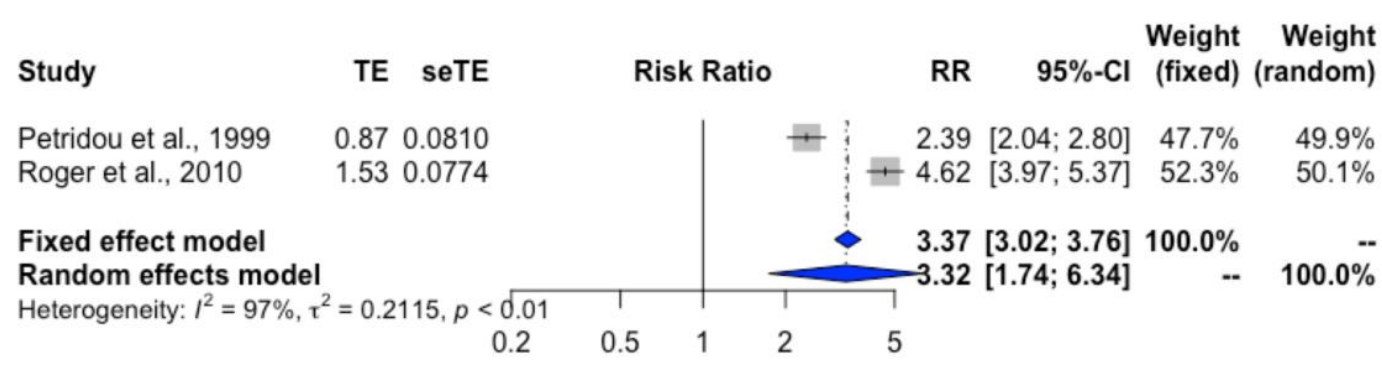

(b) Total protein (g/day)

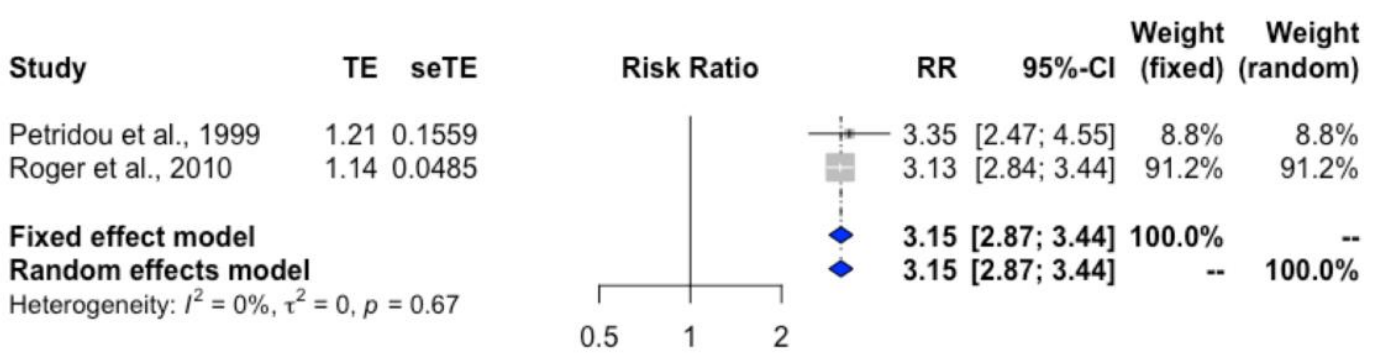

Figure 3. Risk ratio of menarche according to total EI (a) and protein intake (b) among EMO and NEMO girls. Definition of abbreviation: EI, energy intake; EMO, early menarche onset; NEMO, non-early menarche onset; RR: risk ratio.

\subsubsection{Association between Protein Intake and EMO}

The mean difference of protein intake between girls with EMO and NEMO, as expressed by g/day or \%energy, were similar. Given that BMI may be a critical factor in the meta-regression $(p<0.01$; Table S4), subgroup analysis was performed and revealed that among girls with BMI $\geq 18.5 \mathrm{~kg} / \mathrm{m}^{2}$, the higher WMD of protein was observed in the EMO group (WMD $=1.1 \mathrm{~g} / \mathrm{day}, 95 \% \mathrm{CI}=0.5$ to 1.7 , $I^{2}=0$, Figure 4). In addition, high total protein intake was linked to EMO risk ( $R R=3.2,95 \% \mathrm{CI}=2.9$ to $3.4, I^{2}=0 \%$; Figure $3 \mathrm{~b}$ ). Sensitivity analysis also supported the risk of protein intake $[16,21,26]$ to EMO (quartile 3 vs. lowest level, $R R=1.1,95 \% \mathrm{CI}=1.0$ to $1.3, I^{2}=0 \%$; Table S5).

Girls with EMO had higher intake of animal protein [19] than girls with NEMO (MD = 1.4 g/day, $95 \% \mathrm{CI}=0.7$ to $2.1, p<0.001$ ). Additionally, every $1 \mathrm{~g} / \mathrm{d}$ day increase in animal protein intake in early childhood was linked to approximately 2-month earlier AAM (Figure 5) [13,17,24].

\subsubsection{Association between Fat Intake and EMO}

WMD of total fat and MUFAs between EMO and NEMO girls did not show any difference using a random effect model (Table 2). A similar result in total fat consumption could be observed by using either g/day or \%energy. Girls in the EMO group consumed higher PUFAs than NEMO ( $\mathrm{WMD}=0.5 \mathrm{~g} /$ day, $95 \% \mathrm{CI}=0.2$ to $0.8, \mathrm{I}^{2}=21 \%$, Table 2 ). The associations between MUFAs and PUFAs with the EMO risk also revealed the dose-response effects (Figure 6). The higher intake of PUFAs was positively associated with EMO risk when compared with the reference level $(\mathrm{RR}=1.25,95 \% \mathrm{CI}=1.05$ to $1.49, I^{2}=44 \%$, Figure 6 ). In contrast, the higher intake of MUFAs was negatively linked to EMO risk (Figure 6, Table S5) $[16,18,26,27]$. 


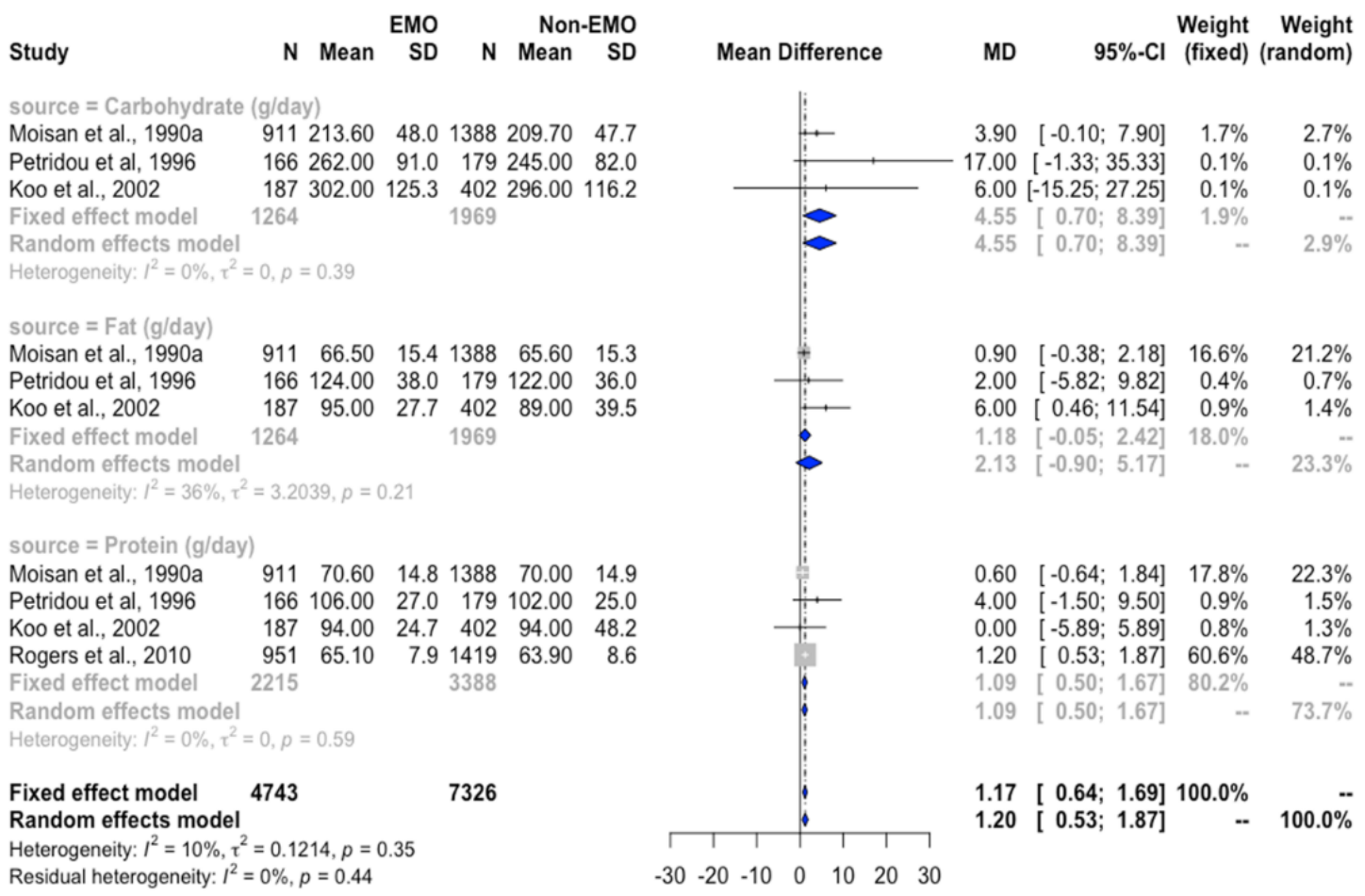

Figure 4. Forest plot of WMD of carbohydrate, protein, and fat intakes between EMO and NEMO groups in healthy girls with $\mathrm{BMI} \geq 18.5 \mathrm{~kg} / \mathrm{m}^{2}$. Definition of abbreviations: $\mathrm{EMO}$, earlier menarche onset; NEMO, non-early menarche onset; MD, mean difference; WMD, weighted mean difference.

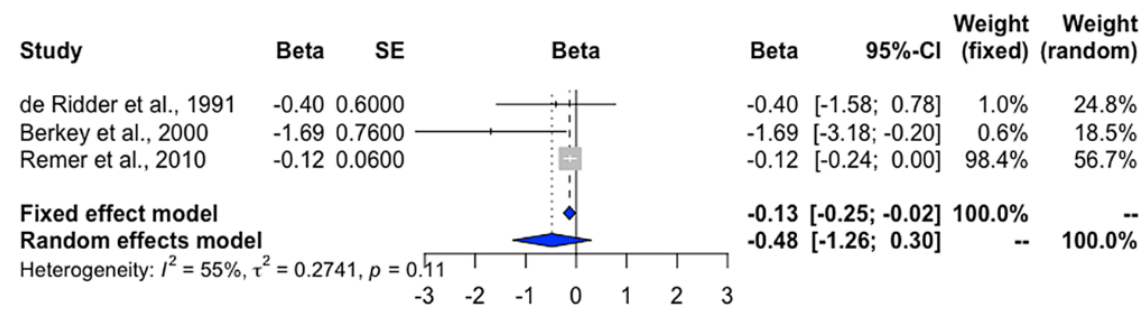

Figure 5. Forest plot of the beta regression coefficient of age at menarche onset for every $1 \mathrm{~g} /$ day increase of animal protein intake. Definition of abbreviations: Beta, beta coefficient; SE, standard error.

\subsubsection{Association between Carbohydrate Intake and EMO}

Similar results in carbohydrate intake could be observed by using either $\mathrm{g} / \mathrm{day}$ or \% energy. However, high heterogeneity was found in the comparison of carbohydrate intake ( $\mathrm{g} /$ day) between the EMO and NEMO groups (Table 2) across the included studies $[12,16,18,27,28]$. In the subgroup analysis, we found that in girls with BMI $\geq 18.5 \mathrm{~kg} / \mathrm{m}^{2}$, the overall WMD of carbohydrate intake was higher in the EMO group than in the NEMO group (WMD $=4.6 \mathrm{~g} /$ day, $95 \% \mathrm{CI}=0.7$ to $8.4, I^{2}=0$, Figure 4). Surprisingly, the higher intake of carbohydrate was linked to later menarche (quartile $2 \mathrm{vs.}$ lowest quartile: $R R=0.81,95 \% C I=0.71-0.94, I^{2}=0 \%$; Figure 6) when considering the adjusted $R R$ from three included studies $[16,21,26]$.

No significant difference was found between fiber intake $[17,18,27]$ and EMO (Table 2). However, Figure 6 reveals that the dose-response effect of fiber intake was likely linked to delay menarche onset (Figure 6). 

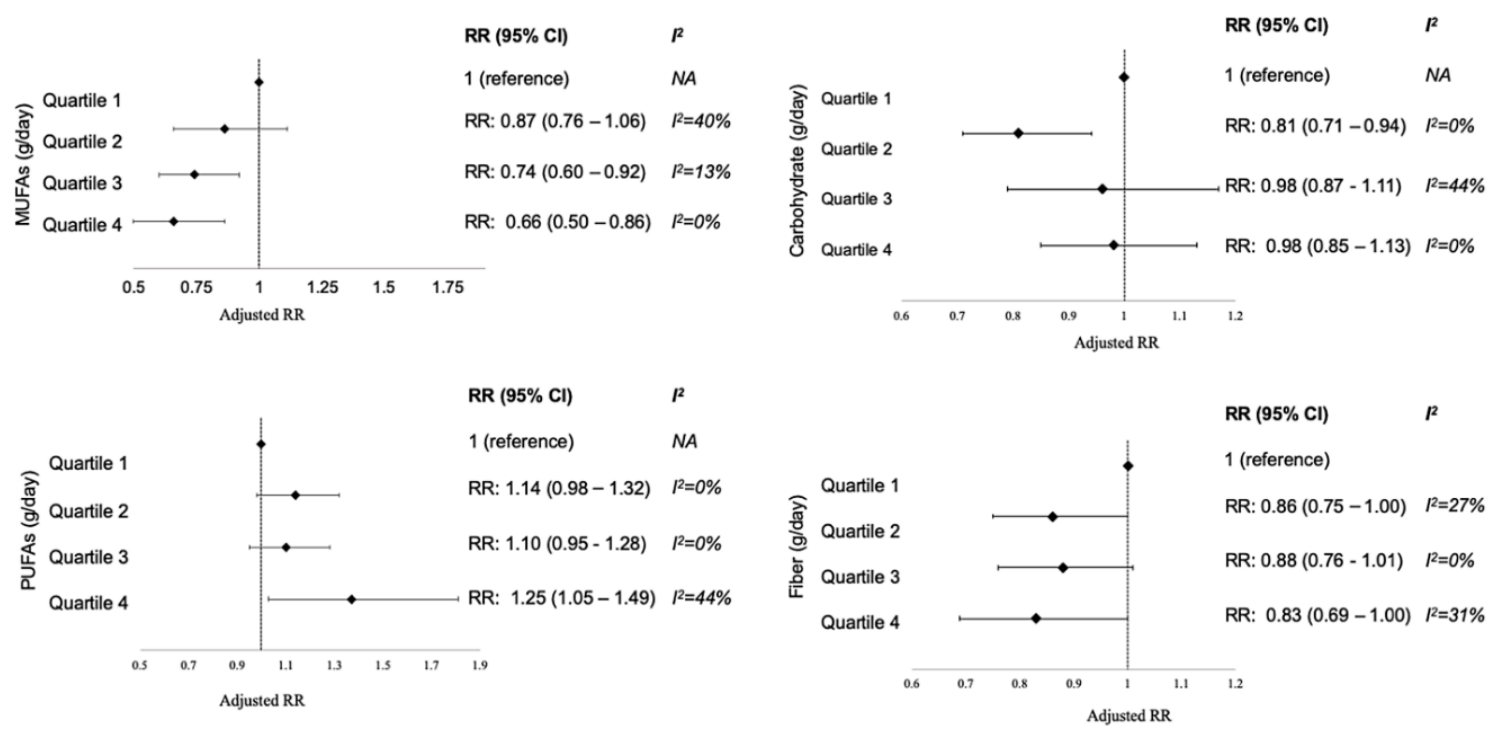

Figure 6. RR of menarche according to quartiles of MUFAs, PUFAs, carbohydrate, and fiber intakes among EMO and NEMO groups. Definition of abbreviations: CI, confidence interval; EMO, earlier menarche onset; MUFAs, monounsaturated fatty acids; NEMO, non-early menarche onset; PUFAs, polyunsaturated fatty acids; RR, risk ratio.

\subsubsection{Association between Micronutrient Intake and EMO}

Girls with EMO were more likely to consume high amounts of magnesium, vitamin C [16,27], and carotene (Table 2). The highest level of iron intake was significantly linked to EMO when compared with the lowest level $\left(R R=1.20,95 \% C I=1.03-1.40, I^{2}=0 \%\right)$, and high intake of vitamin $B_{1}$ was positively associated with EMO $\left(\mathrm{RR}=1.17,95 \% \mathrm{CI}=1.00-1.37, \mathrm{I}^{2}=0 \%\right.$; Table S5).

\section{Discussion}

In this systematic review and meta-analysis, 16 prospective studies involving 10,884 healthy girls were systematically investigated to determine the relationships between nutrient intake in childhood and EMO. High intakes of energy, protein, animal protein, and iron in childhood were associated with EMO. We observed the dose-response effect between PUFA intake and EMO risk and between MUFA intake and delayed menarche onset. In addition, the high intakes of fiber in childhood were likely linked to delayed menarche onset.

The present study confirmed the positive association between EI and EMO in girls with BMI $>18.5 \mathrm{~kg} / \mathrm{m}^{2}$ (Figure S1 and S2), which was not identified in a previous study [1]. The fact that excess calories result in more body fat, which is positively associated with leptin levels mediating pubertal onset [1]. Prepubertal body composition has a critical effect on puberty onset because the conversion of androgen to estrogen occurs in adipose tissue, which is a significant source of extragonadal estrogen $[8,9]$.

Girls who consumed more protein were likely to reach EMO. This may be relevant to the early protein hypothesis which demonstrates that early protein intake predisposes children to adiposity rebound at the time of preceding pubertal marker onset $[13,24]$ that increases the leptin levels [1]. Animal protein intake was negatively associated with AAM (Figure 5). This weak association may be due to the wide range of $95 \% \mathrm{CI}$ and/or the existence of heterogeneity $\left(I^{2}>50 \%\right)$, possibly caused by the different times of dietary assessment among the three included studies $[13,17,24]$ (Table 1 ). Animal protein stimulates insulin-like growth factor 1 (IGF-1) secretion, which causes the expression of gonadotropin-releasing hormone $(\mathrm{GnRH})$, required for pubertal onset [36]. High meat and red meat intake have been associated with EMO in girls [19,34]. Red meat is a major source of zinc, iron, and vitamin $B_{12}$. Rogers et al. [19] found a significant relationship between zinc intake and EMO. 
Higher plasma ferritin status and greater iron storage in middle childhood were also related to earlier AAM [37]. Concordantly, we observed that a high iron intake was associated with EMO. Besides animal protein, vegetable protein has been noticed to be negatively associated with EMO risk [14,17]. Additionally, a delay of AAM was observed when there was a high intake of grains, nuts, beans, legumes, and fruits, which are the primary sources of vegetable protein [16,17].

Fat intake is a strong predictor of earlier AAM, independent of body fat percentage [27]. However, there was a lack of statistical evidence of overall WMD of fat intake in relation to EMO. The fact is that the typical western diet, high in saturated fatty acids (FAs), is largely related to the increased prevalence of obesity, a critical factor which may lead to early puberty [9], and a high-fat diet during puberty may accelerate breast development [38]. Menarche represents the final physical event in a series of steps known as puberty [39] and typically occurs approximately two years after the onset of breast development [40]. Therefore, it is proposed that a high-fat diet may be associated with EMO because it accelerates breast development, the earliest secondary sexual characteristic in girls.

PUFA intake during early childhood has been found to influence puberty onset [41]. The doseresponse effect of PUFA intake during late childhood was positively associated with EMO in the present study. PUFAs are essential FAs involved in the growth and reproductive procress through direct effects on steroidogenic machinery and mammary gland development [41]. In vitro, PUFAs modulate adrenal steroidogenesis and act on steroidogenesis-related transcription factors that affect steroid acute regulator protein expression [42]. Eventually, adrenal androgen stimulates GnRH neurons that are required for puberty onset [1]. Maclure et al. [27] revealed that only n-3 FAs were related to EMO in girls, not n-6 FAs. In contrast, an effect of n-6 PUFAs on puberty onset was discovered [43]. The ratio of n-6 to n-3 FAs is crucial to health. The timing of dietary exposure and the n- 6 to n-3 FA ratio is related to puberty onset [41]. In vivo, an increased ratio of n-6 to n-3 FAs (5:1) modulated the reproductive function in female zebrafish, independent of the total dietary lipid levels [44]. The optimal n-6 to n-3 FA ratio for healthy puberty was beyond our study but merited further investigation.

The effect of MUFA intake during childhood on EMO has been inconsistent. A recent in vitro and in vivo study revealed that oleic acid, a MUFA, and the primary FA in olive oil, could affect puberty onset by stimulating mammary gland development and increasing the serum IGF-1 levels [45]. In contrast, we observed that MUFA intake was likely linked to delayed menarche onset underlying dose-response effects after pooling adjusted risk ratios from three studies conducted in Canada $[16,18,26]$ and one conducted in the United States [27]. In Canada, the main dietary source of MUFA is canola oil, which accounted for three quarters of all processed vegetable oils during the 1990s [46]. Therefore, the type of MUFA intake, which protected girls from EMO in the present study, may be primarily from canola oil. Thus, more studies are warranted to assess the role of specific types of MUFAs on EMO.

A high carbohydrate intake contributes to excess EI and causes substantial weight gain, as stated, eventually influencing puberty onset [47]. In girls with $B M I \geq 18.5 \mathrm{~kg} / \mathrm{m}^{2}$, we observed a high pooled WMD of carbohydrate intake in the EMO group than in the NEMO group, derived from unadjusted analysis. However, the carbohydrate was found to delay menarche onset, as determined using adjusted models (Figure 6). This may be explained by that carbohydrate could conceivably influence estrogen metabolism by depressing the formation of catechol estrogen from estradiol [48]. Remarkably, the overall WMD of carbohydrate intake in girls with EMO was lower than that in girls with NEMO among girls with BMI $<18.5 \mathrm{~kg} / \mathrm{m}^{2}$, as determined from the data of Maclude et al. [27] and Tehrani et al. [28]. Noteworthy, malnutrition was found to be linked to a delay of AAM [49] that may not be solely attributed to the amount of carbohydrate intake.

We found that the dose-response effect of fiber intake in childhood was likely associated with a delayed menarche onset. Fiber intake has been considered to modulate circulating estrogen levels, which affects puberty onset mediated by the hypothalamus-pituitary-gonad system $[1,17]$. A cross-sectional study of 46 countries and areas discovered a strong positive correlation between fiber intake and AAM [50]. Higher intakes of insoluble fiber, cellulose fiber, and grain fiber, which are major sources of dietary fiber, were observed in girls who had later menarche onset [17,18]. Expressed 
in doses, consumption of either 18.19-21.81 g/day of fiber (quartile 1) or more than $25.48 \mathrm{~g} / \mathrm{day}$ of fiber (quartile 4) was significantly linked to delayed menarche onset. Though the consumption of 21.81-25.48 g/day of fiber (quartile 3) did not show a significant association with EMO, it seems to approach the borderline significance $(p=0.07)$. However, Cheng et al. [22] denied the association between fiber intake and AAM, which included much lower doses (7.9-28.3 microgram/day) than that in the included studies $[17,18]$. In fact, the doses of fiber in our meta-analysis are much higher than the recommended level calculated by age plus $5 \mathrm{~g} /$ day [51]. These contrasting findings are believed to be due to Bertrand's rule, which states that either health benefits or adverse consequences are associated with nutrient intake at either optimal intake or beyond this threshold [52]. Furthermore, dietary fiber-rich foods often contain high amounts of isoflavones [23]. Isoflavones are known to have antiestrogen effects because they inhibit the actions of enzyme aromatase and of $17 \beta$-hydroxysteroid dehydrogenase and directly interact with estrogen receptors to limit endogenous estrone and estradiol synthesis [1]. Girls with a high intake of isoflavones entered puberty later [22]. However, soy-based infant formula (containing isoflavones) was not found to be associated with early puberty onset [53]. The timing effect of isoflavones on pubertal development warrants future research.

We found that girls with EMO consumed higher intake of carotene in childhood, which requires a further mechanistic explanation. Carotene was hypothesized in one prospective study to have antiestrogenic effects through inhibition of estrogen signaling via $17 \beta$-estradiol that may protect girls from early puberty onset [54], as previously stated [1]. However, a very high carotene intake reduced fertility in cows [55], indicating the opposing dose-dependent effect [56] of carotene on reproductive function. Although the present study observed the weak association of vitamin $\mathrm{C}$ and magnesium with EMO in limited studies, the precise mechanism needs to be elucidated in further study.

\section{Strengths and Limitations}

This is the first systematic review and meta-analysis to explain the association between EMO and nutrient intake during childhood. First, only studies with a longitudinal design were selected to explore the natural effects of energy and dietary factors on menarche onset. This reduced the possibility of outcome misclassification and reverse causation bias. Second, we carefully explained the effects of nutrient intake on EMO in girls whose BMI was in the normal range $\left(18.5\right.$ to $\left.19.6 \mathrm{~kg} / \mathrm{m}^{2}\right)$ in the included studies due to the fact that malnutrition was associated with a delay in AAM [49]. Third, various statistical methods were employed to ensure the robustness of our findings.

Some limitations of this study are addressed here. The dietary assessment methods differed between studies that limited the comparison of results and admitted measurement errors. Though most studies have controlled similar potential factors, such as mother's AAM, parent education, age, height, body weight, and total EI, we could not contact authors to extract the RR values that were adjusted by the same confounders to attempt higher homogeneity across studies. In addition, we could not control the effect of modifiers of pubertal development, including birth weight [18], physical activity [3], and socioeconomic status [12,26]. Third, with respect to EMO, the recommended nutrient doses based on quartiles of nutrient intake could not be established because of a lack of relevant data. Fourth, we did not investigate the association between dietary quality, food groups, and EMO. However, it is important to identify the nutrients involved in disease etiology in order to isolate the true causative agents, and nutrient-focused research, enhances the mechanistic understanding of food and diet effects on EMO [10]. Finally, our findings may not be generalizable to all ethnicities, especially those in Eastern countries, because most of the selected studies were conducted in Western countries.

\section{Conclusions}

Caloric over-consumption and the association with EMO risk may be contributed by excess protein and carbohydrate intake during childhood in healthy girls. Through a high intake of animal proteins-, iron-, and PUFA-rich foods, girls are more likely to be EMO. In contrast, a high intake of fiber- and MUFA-enriched products is linked to a delay in menarche onset. Forthcoming studies are 
welcome to investigate the role of the specific type of MUFAs, PUFAs, and the n-6:n-3 PUFA ratios on EMO that may promote healthy sexual maturity in girls.

Supplementary Materials: The following are available online at http://www.mdpi.com/2072-6643/12/9/2544/s1, Table S1: PECOS criteria for inclusion and exclusion criteria, Table S2: Quality assessment of the included studies using a modified Quality Assessment Tool for Systematic Reviews of Observational Studies (QATSO) Score for assessing relationship between prepubertal nutrient intake and EMO, Table S3: Analyses of publication bias using Egger Test for assessing relationship between prepubertal nutrient intake and EMO, Table S4: Univariate meta-regression analyses, Table S5: Association between EMO and quartile of nutrient intake in childhood, Figure S1: Forest plot of mean differences of carbohydrate, total fat, and protein intake between EMO and NEMO among girls with BMI $<18.5 \mathrm{~kg} / \mathrm{m}^{2}$, Figure S2: Association between EMO and quartile of nutrient intake in childhood.

Author Contributions: Conceptualization, S.-Y.H. and Y.C.C.; methodology, S.-Y.H. and Y.C.C.; software, H.-Y.F.; validation, N.T.K.N., H.-Y.F., and Q.T.V.H.; formal analysis, N.T.K.N., H.-Y.F., and M.-C.T.; investigation, T.-H.T.; data curation, N.T.K.N., H.-Y.F., and M.-C.T.; writing—original draft preparation, N.T.K.N.; writing-review and editing, S.-Y.H., Y.C.C.; visualization T.-H.T. and Q.T.V.H.; supervision, S.-Y.H., Y.C.C.; funding acquisition, Y.C.C.; All authors have read and agreed to the published version of the manuscript.

Funding: This research was funded by Ministry of Science and Technology, Taiwan, grant number 107-2314-B-532-002-MY3.

Acknowledgments: We thank Yang Chen and Jia-Woei Hou for providing clinical ideas in performing this meta-analysis.

Conflicts of Interest: The authors declare no conflict of interest. The funders had no role in the design of the study; in the collection, analyses, or interpretation of data; in the writing of the manuscript, or in the decision to publish the results.

\section{References}

1. Cheng, G.; Buyken, A.E.; Shi, L.; Karaolis-Danckert, N.; Kroke, A.; Wudy, S.A.; Degen, G.H.; Remer, T. Beyond overweight: Nutrition as an important lifestyle factor influencing timing of puberty. Nutr. Rev. 2012, 70, 133-152. [CrossRef] [PubMed]

2. Must, A.; Phillips, S.M.; Naumova, E.N.; Blum, M.; Harris, S.; Dawson-Hughes, B.; Rand, W.M. Recall of early menstrual history and menarcheal body size: After 30 years, how well do women remember? Am. J. Epidemiol. 2002, 155, 672-679. [CrossRef] [PubMed]

3. Calthorpe, L.; Brage, S.; Ong, K.K. Systematic review and meta-analysis of the association between childhood physical activity and age at menarche. Acta Paediatr. 2019, 108, 1008-1015. [CrossRef] [PubMed]

4. Dossus, L.; Allen, N.; Kaaks, R.; Bakken, K.; Lund, E.; Tjonneland, A.; Olsen, A.; Overvad, K.; Clavel-Chapelon, F.; Fournier, A.; et al. Reproductive risk factors and endometrial cancer: The European Prospective Investigation into Cancer and Nutrition. Int. J. Cancer 2010, 127, 442-451. [CrossRef]

5. Collaborative Group on Hormonal Factors in Breast Cancer. Menarche, menopause, and breast cancer risk: Individual participant meta-analysis, including 118964 women with breast cancer from 117 epidemiological studies. Lancet Oncol. 2012, 13, 1141-1151. [CrossRef]

6. Charalampopoulos, D.; McLoughlin, A.; Elks, C.E.; Ong, K.K. Age at menarche and risks of all-cause and cardiovascular death: A systematic review and meta-analysis. Am. J. Epidemiol. 2014, 180, 29-40. [CrossRef]

7. Jacobsen, B.K.; Heuch, I.; Kvåle, G. Association of low age at menarche with increased all-cause mortality: A 37-year follow-up of 61,319 Norwegian women. Am. J. Epidemiol. 2007, 166, 1431-1437. [CrossRef]

8. Kaplowitz, P.B. Link between body fat and the timing of puberty. Pediatrics 2008, 121, S208-S217. [CrossRef]

9. Chen, Y.-C.; Fan, H.-Y.; Yang, C.; Hsieh, R.-H.; Pan, W.-H.; Lee, Y.L. Assessing causality between childhood adiposity and early puberty: A bidirectional Mendelian randomization and longitudinal study. Metabolism 2019, 100, 153961. [CrossRef]

10. Tapsell, L.C.; Neale, E.P.; Satija, A.; Hu, F.B. Foods, nutrients, and dietary patterns: Interconnections and implications for dietary guidelines. Adv. Nutr. 2016, 7, 445-454. [CrossRef]

11. Meyer, F.; Moisan, J.; Marcoux, D.; Bouchard, C. Dietary and physical determinants of menarche. Epidemiology 1990, 1, 377-381. [CrossRef] [PubMed]

12. Petridou, E.; Syrigou, E.; Toupadaki, N.; Zavitsanos, X.; Willett, W.; Trichopoulos, D. Determinants of age at menarche as early life predictors of breast cancer risk. Int. J. Cancer 1996, 68, 193-198. [CrossRef] 
13. Berkey, C.S.; Gardner, J.D.; Lindsay Frazier, A.; Colditz, G.A. Relation of childhood diet and body size to menarche and adolescent growth in girls. Am. J. Epidemiol. 2000, 152, 446-452. [CrossRef] [PubMed]

14. Gunther, A.L.B.; Karaolis-Danckert, N.; Kroke, A.; Remer, T.; Buyken, A.E. Dietary protein intake throughout childhood is associated with the timing of puberty. J. Nutr. 2010, 140, 565-571. [CrossRef]

15. Cheng, H.L.; Raubenheimer, D.; Steinbeck, K.; Baur, L.; Garnett, S. New insights into the association of mid-childhood macronutrient intake to pubertal development in adolescence using nutritional geometry. Br. J. Nutr. 2019, 122, 274-283. [CrossRef]

16. Moisan, J.; Meyer, F.; Gingras, S. Diet and age at menarche. Cancer Causes Control. 1990, 1, 149-154. [CrossRef]

17. De Ridder, C.M.; Thijssen, J.H.; Van't Veer, P.; van Duuren, R.; Bruning, P.F.; Zonderland, M.L.; Erich, W.B. Dietary habits, sexual maturation, and plasma hormones in pubertal girls: A longitudinal study. Am. J. Clin. Nutr. 1991, 54, 805-813. [CrossRef]

18. Koo, M.M.; Rohan, T.E.; Jain, M.; McLaughlin, J.R.; Corey, P.N. A cohort study of dietary fibre intake and menarche. Public Health Nutr. 2002, 5, 353-360. [CrossRef]

19. Rogers, I.S.; Northstone, K.; Dunger, D.B.; Cooper, A.R.; Ness, A.R.; Emmett, P.M. Diet throughout childhood and age at menarche in a contemporary cohort of British girls. Public Health Nutr. 2010, 13, 2052-2063. [CrossRef]

20. Merzenich, H.; Boeing, H.; Wahrendorf, J. Dietary fat and sports activity as determinants for age at menarche. Am. J. Epidemiol. 1993, 138, 217-224. [CrossRef]

21. Koprowski, C.; Ross, R.K.; Mack, W.J.; Henderson, B.E.; Bernstein, L. Diet, body size and menarche in a multiethnic cohort. Br. J. Cancer 1999, 79, 1907. [CrossRef] [PubMed]

22. Cheng, G.; Remer, T.; Prinz-Langenohl, R.; Blaszkewicz, M.; Degen, G.H.; Buyken, A.E. Relation of isoflavones and fiber intake in childhood to the timing of puberty. Am. J. Clin. Nutr. 2010, 92, 556-564. [CrossRef] [PubMed]

23. Grace, P.B.; Taylor, J.I.; Low, Y.-L.; Luben, R.N.; Mulligan, A.A.; Botting, N.P.; Dowsett, M.; Welch, A.A.; Khaw, K.-T.; Wareham, N.J.; et al. Phytoestrogen concentrations in serum and spot urine as biomarkers for dietary phytoestrogen intake and their relation to breast cancer risk in European prospective investigation of cancer and nutrition-norfolk. Cancer Epidemiol. Biomarkers Prev. 2004, 13, 698-708. [PubMed]

24. Remer, T.; Shi, L.; Buyken, A.E.; Maser-Gluth, C.; Hartmann, M.F.; Wudy, S.A. Prepubertal adrenarchal androgens and animal protein intake independently and differentially influence pubertal timing. J. Clin. Endocrinol. Metab. 2010, 95, 3002-3009. [CrossRef] [PubMed]

25. Wang, Y. Is obesity associated with early sexual maturation? A comparison of the association in American boys versus girls. Pediatrics 2002, 110, 903-910. [CrossRef]

26. Moisan, J.; Meyer, F.; Gingras, S. A nested case-control study of the correlates of early menarche. Am. J. Epidemiol. 1990, 132, 953-961. [CrossRef]

27. Maclure, M.; Travis, L.B.; Willett, W.; MacMahon, B. A prospective cohort study of nutrient intake and age at menarche. Am. J. Clin. Nutr. 1991, 54, 649-656. [CrossRef]

28. Tehrani, F.R.; Moslehi, N.; Asghari, G.; Gholami, R.; Mirmiran, P.; Azizi, F. Intake of dairy products, calcium, magnesium, and phosphorus in childhood and age at menarche in the Tehran Lipid and Glucose Study. PLOS ONE 2013, 8, e57696. [CrossRef]

29. Wong, W.C.; Cheung, C.S.; Hart, G.J. Development of a quality assessment tool for systematic reviews of observational studies (QATSO) of HIV prevalence in men having sex with men and associated risk behaviours. Emerg. Themes Epidemiol. 2008, 5, 23. [CrossRef]

30. Hozo, S.P.; Djulbegovic, B.; Hozo, I. Estimating the mean and variance from the median, range, and the size of a sample. BMC Med. Res. Methodol. 2005, 5, 13. [CrossRef]

31. Higgins, J.P.T.; Thompson, S.G.; Deeks, J.J.; Altman, D.G. Measuring inconsistency in meta-analyses. BMJ 2003, 327, 557-560. [CrossRef] [PubMed]

32. Hayashino, Y.; Noguchi, Y.; Fukui, T. Systematic evaluation and comparison of statistical tests for publication bias. J. Epidemiol. 2005, 15, 235-243. [CrossRef] [PubMed]

33. Song, F.; Khan, K.S.; Dinnes, J.; Sutton, A.J. Asymmetric funnel plots and publication bias in meta-analyses of diagnostic accuracy. Int. J. Epidemiol. 2002, 31, 88-95. [CrossRef] [PubMed]

34. Kissinger, D.G.; Sanchez, A. The association of dietary factors with the age of menarche. Nutr. Res. 1987, 7, 471-479. [CrossRef] 
35. Farahmand, M.; Tehrani, F.R.; Amiri, P.; Azizi, F. Barriers to healthy nutrition: Perceptions and experiences of Iranian women. BMC Public Health 2012, 12, 1064. [CrossRef]

36. Thankamony, A.; Ong, K.K.; Ahmed, M.L.; Ness, A.R.; Holly, J.M.P.; Dunger, D.B. Higher levels of IGF-I and adrenal androgens at age 8 years are associated with earlier age at menarche in girls. J. Clin. Endocrinol. Metab. 2012, 97, E786-E790. [CrossRef]

37. Villamor, E.; Marín, C.; Mora-Plazas, M.; Oliveros, H. Micronutrient status in middle childhood and age at menarche: Results from the Bogotá School Children Cohort. Br. J. Nutr. 2017, 118, 1097-1105. [CrossRef]

38. Olson, L.K.; Tan, Y.; Zhao, Y.; Aupperlee, M.D.; Haslam, S.Z. Pubertal exposure to high fat diet causes mouse strain-dependent alterations in mammary gland development and estrogen responsiveness. Int. J. Obes. 2010, 34, 1415-1426. [CrossRef]

39. Maher, J.Y.; Zacur, H.A. Menarche/menopause. In Encyclopedia of Reproduction; Elsevier: Amsterdam, The Netherlands, 2018; pp. 245-249.

40. Valeggia, C.R.; Núñez-de la Mora, A. Human reproductive ecology. In Basics in Human Evolution; Elsevier: Amsterdam, The Netherlands, 2015; pp. 295-308.

41. Anderson, B.M.; MacLennan, M.B.; Hillyer, L.M.; Ma, D.W.L. Lifelong exposure to n-3 PUFA affects pubertal mammary gland development. Appl. Physiol. Nutr. Metab. 2014, 39, 699-706. [CrossRef]

42. Sampath, H.; Ntambi, J.M. Polyunsaturated fatty acid regulation of genes of lipid metabolism. Annu. Rev. Nutr. 2005, 25, 317-340. [CrossRef]

43. Hilakivi-Clarke, L.; Clarke, R.; Onojafe, I.; Raygada, M.; Cho, E.; Lippman, M. A maternal diet high in n-6 polyunsaturated fats alters mammary gland development, puberty onset, and breast cancer risk among female rat offspring. Proc. Natl. Acad. Sci. USA 1997, 94, 9372-9377. [CrossRef] [PubMed]

44. Fowler, L.A.; Dennis-Cornelius, L.N.; Dawson, J.A.; Barry, R.J.; Davis, J.L.; Powell, M.L.; Yuan, Y.; Williams, M.B.; Makowsky, R.; D'Abramo, L.R.; et al. Both dietary ratio of n-6 to n-3 fatty acids and total dietary lipid are positively associated with adiposity and reproductive health in zebrafish. Curr. Dev. Nutr. 2020, 4, nzaa034. [CrossRef] [PubMed]

45. Meng, Y.; Zhang, J.; Yuan, C.; Zhang, F.; Fu, Q.; Su, H.; Zhu, X.; Wang, L.; Gao, P.; Shu, G.; et al. Oleic acid stimulates HC11 mammary epithelial cells proliferation and mammary gland development in peripubertal mice through activation of CD36-Ca2+ and PI3K/Akt signaling pathway. Oncotarget 2018, 9, 12982. [CrossRef] [PubMed]

46. C.C.O. Canada Canola Oil: Nutritional Properties. Available online: https://www.canolacouncil.org/media/ 515159/canola_oil_nutritional_properties.pdf (accessed on 7 November 2019).

47. Li, W.; Liu, Q.; Deng, X.; Chen, Y.; Liu, S.; Story, M. Association between obesity and puberty timing: A systematic review and meta-analysis. Int. J. Environ. Res. Public Health 2017, 14, 1266. [CrossRef]

48. Anderson, K.E.; Kappas, A.; Conney, A.H.; Bradlow, H.L.; Fishman, J. The influence of dietary protein and carbohydrate on the principal oxidative biotransformations of estradiol in normal subjects. J. Clin. Endocrinol. Metab. 1984, 59, 103-107. [CrossRef] [PubMed]

49. Leenstra, T.; Petersen, L.T.; Kariuki, S.K.; Oloo, A.J.; Kager, P.A.; Ter Kuile, F.O. Prevalence and severity of malnutrition and age at menarche; cross-sectional studies in adolescent schoolgirls in western Kenya. Eur. J. Clin. Nutr. 2005, 59, 41-48. [CrossRef]

50. Hughes, R.E.; Jones, E. Intake of dietary fibre and the age of menarche. Ann. Hum. Biol. 1985, 12, 325-332. [CrossRef]

51. Williams, C.L. Importance of dietary fiber in childhood. J. Am. Diet. Assoc. 1995, 95, 1140-1149. [CrossRef]

52. Hayes, D.P. Adverse effects of nutritional inadequacy and excess: A hormetic model. Am. J. Clin. Nutr. 2008, 88, 578S-581S. [CrossRef]

53. Sinai, T.; Ben-Avraham, S.; Guelmann-Mizrahi, I.; Goldberg, M.R.; Naugolni, L.; Askapa, G.; Katz, Y.; Rachmiel, M. Consumption of soy-based infant formula is not associated with early onset of puberty. Eur. J. Nutr. 2019, 58, 681-687. [CrossRef]

54. Eliassen, A.H.; Liao, X.; Rosner, B.; Tamimi, R.M.; Tworoger, S.S.; Hankinson, S.E. Plasma carotenoids and risk of breast cancer over $20 \mathrm{y}$ of follow-up. Am. J. Clin. Nutr. 2015, 101, 1197-1205. [CrossRef] [PubMed] 
55. Folman, Y.; Ascarelli, I.; Kraus, D.; Barash, H. Adverse effect of $\beta$-carotene in diet on fertility of dairy cows. J. Dairy Sci. 1987, 70, 357-366. [CrossRef]

56. Boeing, H. Nutritional epidemiology: New perspectives for understanding the diet-disease relationship? Eur. J. Clin. Nutr. 2013, 67, 424-429. [CrossRef] [PubMed] 